\title{
ON THE SPECTRA OF THE RESTRICTIONS OF AN OPERATOR
}

\author{
BY
}

\author{
DOMINGO A. HERRERO
}

\begin{abstract}
Let $T$ be a bounded linear operator from a complex Banach space $\mathcal{X}$ into itself and let $\Re$ be a closed invariant subspace of $T$. Let $T \mid \vartheta \mathbb{R}$ denote the restriction of $T$ to $\mathscr{T}$ and let $\sigma$ denote the spectrum of an operator. The main results say that: (1) If $\mathcal{X}$ is the closed linear span of a family $\left\{\pi_{r}\right\}$ of invariant subspaces, then every component of $\sigma(T)$ intersects the closure of the set $U_{v} \sigma\left(T \mid T_{r}\right)$ and every point of $\sigma(T) \backslash$ $\cup, \sigma\left(T \mid \pi_{p}\right)$ is an approximate eigenvalue of $T$. (2) If $\mathcal{X}$ is the closed linear span of a finite family $\left\{\Re_{1}, \ldots, \Re_{n}\right\}$ of invariant subspaces, and the spectra $\sigma\left(T \mid \Re_{j}\right), j=1,2, \ldots, n$, are pairwise disjoint, then $\mathcal{X}$ is actually equal to the algebraic direct sum of the $\pi_{\text {; }} s$, the $\Re_{\text {, }}$ 's are hyperinvariant subspaces of $T$ and $\sigma(T)=\bigcup_{j=1}^{n} \sigma\left(T \mid \Re_{j}\right)$. This last result is sharp in a certain specified sense. The results of (1) have a "dual version" (1); (1) and (1) are applied to analyze the spectrum of an operator having a chain of invariant subspaces which is "piecewise well-ordered by inclusion", extending in several ways recent results of J. D. Stafney on the spectra of lower triangular matrices.
\end{abstract}

1. On the spectra of the restrictions of $T \in \mathcal{L}(\mathfrak{X})$. In what follows $\mathfrak{X}$ will denote a Banach space over the complex field C: Operator and subspace will mean bounded linear map from $\mathcal{X}$ into $\mathcal{X}$ and closed linear manifold, respectively. The Banach algebra of all operators acting on $\mathscr{X}$ will be denoted by $\mathcal{L}(\mathcal{X})$. Let $\mathfrak{T} \in$ Lat $T$, the lattice of invariant subspaces of an operator $T$. Here we shall study the relations between the spectrum of $T$ and the spectra of $T \mid \Re$ (the restriction of $T$ to $\Re$ thought as an operator acting on $\Re$ ) and $\bar{T}_{\mathscr{T}} \in \mathcal{L}(\mathcal{X} / \mathscr{T})$, the operator induced by $T$ on the quotient space $\mathcal{X} / \mathscr{T}$, which is defined by the equality $\bar{T}_{\pi x}=\pi T x$, for all $x \in \mathcal{X}$, where $\pi$ : $\mathcal{X} \rightarrow \mathcal{X} / \mathfrak{R}$ is the canonical projection.

Let $\sigma(T)$ be the spectrum of $T \in \mathcal{L}(\mathcal{X})$; following P. R. Halmos [3], we shall denote by $\sigma_{\mathrm{ap}}(T)$ the approximate point spectrum of $T$, i.e. $\sigma_{\mathrm{ap}}(T)=\{\lambda$ $\in \sigma(T)$ : there exists a sequence $\left(x_{n}\right)_{n=1}^{\infty}$ of vectors of norm 1 such that

Received by the editors July 30, 1973.

AMS (MOS) subject classifications (1970). Primary 47A10, 47A15; Secondary 47A60.

Key words and phrases. Riesz' functional calculus, invariant subspace, hyperinvariant subspace, spanning set of subspaces, spectrum, restriction of an operator, spectra of the restrictions, disjointness, component, algebraic sum, algebraic direct sum, operator induced on the quotient space, spectral radius, Hilbert's theorem on approximation of Jordan curves by lemniscates. 
$\left\|(T-\lambda) x_{n}\right\| \rightarrow 0$, as $\left.n \rightarrow \infty\right\}$. It is well known that $\sigma_{\text {ap }}(T)$ contains $\sigma_{p}(T)$, the point spectrum of $T, \sigma_{\mathrm{ap}}(T)$ is a closed subset of $\sigma(T), \sigma_{\mathrm{ap}}(T) \supset \partial \sigma(T)$, (where $\partial K$ denotes the boundary of a set $K \subset \mathbf{C}$ ) and $(T-\lambda)$ is a semiFredholm operator of negative index for all $\lambda \in \sigma(T) \backslash \sigma_{\mathrm{ap}}(T)$. If $\Re \in$ Lat $T$, then $\partial \sigma(T \mid \Re) \subset \sigma_{\text {ap }}(T \mid \Re) \subset \sigma_{\text {ap }}(T)$; in particular, $\sigma(T \mid \Re) \cap \sigma(T)$ $\neq \varnothing$, unless $\mathscr{T}=\{0\}$ (in which case $\sigma(T \mid \Re)=\varnothing$ ). If $T^{*} \in \mathcal{L}\left(X^{*}\right)$ denotes the adjoint of $T$ (acting on the dual space $X^{*}$ of $\mathscr{X}$ ), then $\sigma\left(T^{*}\right)=\sigma(T)$ (except when $\mathcal{X}$ is a Hilbert space and $T^{*}$ is defined "via inner product", in which case the above equality is replaced by $\left.\sigma\left(T^{*}\right)=\{\bar{\lambda}: \lambda \in \sigma(T)\}\right)$. The reader is referred to [1], [3], [9], [10] for details.

A fundamental tool for this paper is the Riesz functional calculus: if $f$ is a function analytic in a neighborhood $\Omega$ of $\sigma(T)$ and $\gamma$ is a suitably oriented finite family of rectifiable closed pairwise disjoint Jordan curves such that $(1 / 2 \pi i) \int_{\gamma}(\lambda-z)^{-1} d \lambda=1$ for all $z \in \sigma(T)$, then $f(T)=(1 / 2 \pi i) \int_{\gamma} f(\lambda)(\lambda-$ $T)^{-1} d \lambda$ defines an operator on $\mathcal{X}$ which can be approximated in the norm by rational function of $T$, with poles outside $\sigma(T)$; then $f(T) \in \mathbb{Q}_{T}^{a}$, the analytic algebra generated by $T$. Moreover, if $f$ is actually analytic in a neighborhood of $\hat{\sigma}(T)=\mathbf{C} \backslash \rho_{\infty}(T)$, where $\rho_{\infty}(T)$ denotes the unbounded component of the resolvent set $\rho(T)=\mathbf{C} \backslash \sigma(T)$ of $T$, then $f(T) \in \mathbb{Q}_{T}$, the weak closure of the polynomials in $T$. (This is an easy consequence of Runge's theorem; see [6]. The Riesz' functional calculus that we need is contained in [9] and [10, Chapter XI].) We shall extensively use a particular case of this functional calculus; namely, if $\sigma(T)=\sigma_{0} \cup \sigma_{1}$, where $\sigma_{0}$ and $\sigma_{1}$ are nonempty disjoint clopen (i.e., closed and open) subsets of $\sigma(T)$ and $f$ is defined to be identically zero in a neighborhood of $\sigma_{0}$ and identically one in a neighborhood of $\sigma_{1}$, then $f(T)$ is an idempotent element of $\mathbb{Q}_{T}^{a}$ such that $\chi=\operatorname{ker} f(T) \oplus \operatorname{ran} f(T)$ (the algebraic direct sum of the kernel and the range of $f(T)), \sigma(T \mid \operatorname{ker} f(T))=\sigma_{0}$ and $\sigma(T \mid \operatorname{ran} f(T))=\sigma_{1} . f(T)$ will be called the idempotent associated to $\sigma_{1}$.

THEOREM 1. Let $\left\{\Re_{\nu}: \nu \in \Phi\right\}$ be an arbitrary family of invariant subspaces of the operator $T$ whose closed linear span $\bigvee\left\{\Re_{\nu}: \nu \in \Phi\right\}$ is the whole space $\mathcal{X}$. Let $\sigma^{\prime}=\cup_{\nu} \sigma\left(T_{\nu}\right)$, where $T_{\nu}=T \mid \Re_{\nu}$ is the restriction of $T$ to $\Re_{\nu}$, and $\sigma=$ closure $\sigma^{\prime}$. Then:

(i) $\sigma(T) \backslash \sigma^{\prime} \subset \sigma_{\text {ap }}(T)$.

(ii) Every clopen subset of $\sigma(T)$ intersects $\sigma^{\prime}$.

(iii) Every component of $\sigma(T)$ intersects $\sigma$.

(iv) In particular, if $\sigma(T)$ is totally disconnected, then $\sigma(T)=\sigma$.

Proof. (i) We proceed as in [5, Theorem 6]: if $\lambda \in \sigma(T) \backslash \sigma^{\prime}$ then (T-

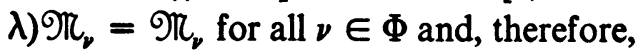

$$
(T-\lambda) \mathcal{X}=(T-\lambda) \bigvee \mathscr{R}_{\nu} \supset(T-\lambda) \sum_{\nu} \mathscr{R}_{\nu}=\sum_{\nu}(T-\lambda) \Re_{\nu}=\sum_{\nu} \mathscr{R}_{\nu},
$$


which is dense in $\mathcal{X}$, i.e. $T-\lambda$ has a dense range, whence the result follows (see $[9$, Chapter IV]).

(ii) Let $\sigma_{0}$ be a clopen subset of $\sigma(T)$ which does not intersect $\sigma^{\prime}$. For fixed $\nu$, let $f_{v}$ be a function analytic in a neighborhood of $\sigma(T) \cup \sigma\left(T_{\nu}\right)$ such that $f(z) \equiv 0$ in a neighborhood of $\sigma_{0}$ and $f(z) \equiv 1$ in a neighborhood of $\sigma(T) \cup$ $\sigma\left(T_{v}\right) \backslash \sigma_{0}$, and let $E_{v}=f_{\nu}(T)$. Observe that $E=E_{\nu}$ is an idempotent independent of $\nu \in \Phi\left[10\right.$, Chapter XI]; in fact, for any $\nu, \mathcal{X}=\operatorname{ran} E_{\nu} \oplus \operatorname{ker} E_{\nu}$ is the decomposition of $\mathcal{X}$ associated to the partition $\sigma(T)=\left[\sigma(T) \backslash \sigma_{0}\right] \cup \sigma_{0}$, where $\sigma\left(T \mid \operatorname{ran} E_{\nu}\right)=\sigma(T) \backslash \sigma_{0}$ and $\sigma\left(T \mid \operatorname{ker} E_{\nu}\right)=\sigma_{0}$, ran $E_{\nu}$ and ker $E_{\nu}$ are obviously invariant under $E_{v}$, and $E_{v}\left|\operatorname{ran} E_{v}=I\right| \operatorname{ran} E_{v}$ (where $I$ denotes the identity operator on $\mathcal{X}$ ) and $E_{\nu} \mid \operatorname{ker} E_{v}=0$. Clearly, the above partition does not depend on $\nu$.

On the other hand, $\Re_{v}$ is invariant under $E$ and $E\left|\Re_{v}=f_{v}(T)\right| \Re_{v}=$ $f_{\nu}\left(T \mid \Re_{\nu}\right)=I \mid \Re_{\nu}$ because $f_{\nu}(z) \equiv 1$ in a neighborhood of $\sigma\left(T_{v}\right)$. Since $\mathcal{X}=\bigvee_{\nu} \mathfrak{T}_{\nu}$, it follows that $E=I$; i.e. ker $E=\{0\}$, which is impossible unless $\sigma_{0}=\varnothing$. This proves (ii).

(iii) Since $\sigma(T)$ is a compact Hausdorff space, every component of $\sigma(T)$ is the intersection of all the clopen subsets containing it. Let $\sigma_{1}=\bigcap\left\{\sigma_{\alpha}\right.$ : $(\alpha \in \Psi) \sigma_{\alpha}$ is clopen and contains $\left.\sigma_{1}\right\}$ be a component of $\sigma(T)$. According to (ii), $\sigma_{\alpha} \cap \sigma^{\prime} \neq \varnothing$ for every $\alpha \in \Psi$. Therefore $\left\{\sigma_{\alpha} \cap \sigma: \alpha \in \Psi\right\}$ is a family of closed subsets of $\sigma(T)$ having the finite intersection property. By compactness, it is clear that $\sigma_{1} \cap \sigma=\cap\left\{\sigma_{\alpha} \cap \sigma: \alpha \in \Psi\right\}$ cannot be empty.

(iv) The proof of (ii) shows that if $\sigma(T)$ is totally disconnected, then $\sigma(T) \subset \sigma$. Now, according to [1], for each $\nu \in \Phi, \sigma\left(T_{\nu}\right) \subset \hat{\sigma}(T)$. Since $\sigma(T)$ is totally disconnected, $\sigma(T)=\hat{\sigma}(T)$ and therefore $\sigma\left(T_{\nu}\right) \subset \sigma(T)$; a fortiori, $\sigma \subset \sigma(T)$.

EXAMPLE $C$ of [5] shows that, in general, $\sigma(T) \not \partial \sigma$. Our next example shows that $\sigma(T) \not \subset \hat{\sigma}$ in the general case; moreover, it also shows that $\sigma$ could be surprisingly small in comparison with $\sigma(T)$, even in the case when $\mathcal{X}$ is the closed span of only two subspaces. The ingredients for the construction of this example were taken from a paper of T. B. Hoover [8].

EXAMPLE E. Let $\mathfrak{X}=\mathfrak{X}_{0} \oplus \mathfrak{X}_{1}$, where $\mathfrak{X}_{0}$ and $\mathfrak{X}_{1}$ are separable infinite dimensional Hilbert spaces. We can write $\mathfrak{X}_{0}=\bigoplus_{n+1}^{\infty} \mathfrak{X}_{0(n)}, X_{1}=$ $\bigoplus_{n=1}^{\infty} \mathscr{X}_{1(n)}$ (all the direct sums considered here are closed orthogonal direct sums in Hilbert spaces), where $\mathscr{X}_{O(n)} \doteq \mathbf{C}^{n} \simeq \mathscr{X}_{1(n)}$, for $n=1,2, \ldots$ Let $\left\{x_{1}^{n}, \ldots, x_{n}^{n}\right\}$ and $\left\{y_{1}^{n}, \ldots, y_{n}^{n}\right\}$ be orthonormal bases of $\mathscr{X}_{0(n)}$ and $\mathscr{X}_{1(n)}$, resp. $(n=1,2, \ldots)$ and define $T \in \mathcal{L}(\mathcal{X})$ as follows: $T=T_{0} \oplus T_{1}, T_{j}=$ $\bigoplus_{n=1}^{\infty} T_{j(n)} \in \mathcal{L}\left(\mathfrak{X}_{j}\right), j=0,1$, where $T_{j(n)}(j=0,1 ; n=1,2, \ldots)$ are the operators acting on $\mathscr{X}_{j(n)}$ defined by $T_{0(n)} x_{1}^{n}=0, T_{0(n)} x_{k}^{n}=x_{k-1}^{n}$, for $k=2$, $3, \ldots, n$, and $T_{1(n)} y_{1}^{n}=0, T_{1(n)} y_{k}^{n}=(1 / n) y_{k-1}^{n}$, for $k=2,3, \ldots, n$.

Let $\mathscr{X}_{2}=\bigoplus_{n=1}^{\infty} \mathscr{X}_{2(n)}$, where $\mathscr{X}_{2(n)}$ is the subspace spanned by the 
orthonormal set $\left\{z_{k}^{n}=c_{k, n}\left(n^{-k} x_{k}^{n}+y_{k}^{n}\right)\right\}_{k=1}^{n}\left(c_{k, n}=\left(1+n^{-2 k}\right)^{-1 / 2}\right)$. It is easy to check that $\mathscr{X}_{0(n)}, \mathscr{X}_{1(n)}, \mathscr{X}_{2(n)} \in$ Lat $T_{0(n)} \oplus T_{1(n)}$, and therefore $\mathscr{X}_{0}, \mathscr{X}_{1}, \mathscr{X}_{2} \in$ Lat $T$. Clearly, we have $\mathscr{X}_{1(n)}+\mathscr{X}_{2(n)}=\mathscr{X}_{0(n)} \oplus \mathscr{X}_{1(n)}$ and, therefore, $\mathscr{X}=\mathscr{X}_{1} \vee \mathscr{X}_{2}$. On the other hand, $\mathscr{X}_{1} \cap \mathscr{X}_{2}=\{0\}$ and $\sigma\left(T \mid \mathscr{X}_{1}\right)$ $=\sigma\left(T \mid \mathscr{X}_{2}\right)=\{0\}$, while $\sigma(T)=D^{-}$(i.e., the closure of the open unit disc; see [3], [8]).

Remarks. (a) Let $\mathbb{Q}_{T}^{\prime}=\{L \in \mathfrak{L}(\mathfrak{X}): L T=T L\}$ be the commutant of $T$ in $\mathcal{L}(X)$ and let $\mathbb{Q}_{T}^{\prime \prime}$ (similarly defined) be the commutant of $\mathbb{Q}_{T}^{\prime}$, i.e. the double commutant of $T$. Then [6] $\mathbb{Q}_{T} \subset \mathbb{Q}_{T}^{a} \subset \mathbb{Q}_{T}^{\prime \prime} \subset \mathbb{Q}_{T}^{\prime}$ and the corresponding lattices of invariant subspaces satisfy the reverse inclusions, i.e.: Lat $\mathbb{Q}_{T}=$ Lat $T \supset$ Lat $\mathbb{Q}_{T}^{a}$ (analytically invariant subspaces) $\supset$ Lat $\mathbb{Q}_{T}^{\prime \prime}$ (bi-invariant subspaces) $\supset$ Lat $\mathbb{Q}_{T}^{\prime}$ (hyperinvariant subspaces) (Lat $\mathbb{Q}_{T}^{\prime}$ has also been studied in [2]). A straightforward computation shows that the operator of Example E satisfies $\mathbb{Q}_{T}=\mathbb{Q}_{T}^{\prime \prime}$ and, therefore, $\mathfrak{X}_{1}, \mathfrak{X}_{2} \in$ Lat $\mathbb{Q}_{T}^{\prime \prime}$. We do not know any example of that kind of pathological behaviour with hyperinvariant $\mathscr{X}_{1}$ and $\mathscr{X}_{2}$.

(b) The arguments of the proof of Theorem 1 can be used to improve a result of T. B. Hoover [8]: If $A \in \mathcal{L}(\mathcal{X}), B \in \mathcal{L}(\mathcal{Y})$ and there exists a quasi-invertible continuous linear map $S: \mathcal{X} \rightarrow \mathcal{O}$ (i.e. ker $S=\{0\}$ and $\operatorname{ran} S$ is dense in $\mathscr{Y}$ ) such that $S A=B S$, then every component of $\sigma(A)$ intersects some component of $\sigma(B)$ and conversely. In fact, the equality $S A=B S$ implies that $S(A-\lambda)^{-1}=(B-\lambda)^{-1} S$ for all $\lambda \in \rho(A) \cap \rho(B)$; thus, if $f$ is a function analytic in a neighborhood of $\sigma(A) \cup \sigma(B)$ which only takes the values 0 and 1 , then $S f(A)=f(B) S$. Since $S$ is quasi-invertible, we conclude that $f(A)=0$ if and only $f(B)=0$, and it clearly follows that every clopen subset of $\sigma(A)$ intersects some clopen subset of $\sigma(B)$. By proceeding as in the proof of (iii), it is easy to see that the clopen subsets can be replaced by components of $\sigma(A)$ and $\sigma(B)$. A minor modification of the example given in [8] shows that $\sigma(A)$ can have exactly one component while $\sigma(B)$ has uncountably many.

Our next result is the promised "dual version" of Theorem 1.

THEOREM $1^{*}$. Let $\left\{\Re_{r}: \nu \in \Phi\right\}$ be an arbitrary family of invariant subspaces of the operator $T$ such that $\cap\left\{\mathcal{X}_{\nu}: \nu \in \Phi\right\}=\{0\}$. Let $\sigma^{\prime}=U_{\nu} \sigma\left(\bar{T}_{\nu}\right)$, where $\bar{T}_{\nu}$ is the operator induced by $T$ on $\mathcal{X} / \mathscr{N}_{\nu}$, and $\sigma=$ closure $\sigma^{\prime}$. Then:

$\left(i^{*}\right) \sigma\left(T^{*}\right) \backslash \sigma^{\prime} \subset \sigma_{\mathrm{ap}}\left(T^{*}\right)$.

(ii*) Every clopen subset of $\sigma(T)$ intersects $\sigma^{\prime}$.

(iii*) Every component of $\sigma(T)$ intersects $\sigma$.

(iv*) If $\sigma(T)$ is totally disconnected, then $\sigma(T)=\sigma$.

Proof. (i*) Let $\Re \in$ Lat $T$ and let $\Re^{\perp}=\left\{x^{*} \in X^{*}\right.$ : ker $\left.x^{*} \supset \Re\right\}$ be the annihilator of $\mathfrak{N}$. It is well known that $\mathfrak{N}^{\perp}$ is a $w^{*}$-closed subspace of 
$\mathcal{X}$ invariant under $T^{*}$, which can be canonically identified with $(\mathcal{X} / \mathscr{T})^{*}$; $T^{*} \mid \Re^{\perp}$ can be identified with $\bar{T}^{*}$ (where, as usual, $\bar{T}$ denotes the operator induced by $T$ on $X / \Re)$ and, therefore, $\sigma\left(T^{*} \mid \Re^{\perp}\right)=\sigma(\bar{T})$ (see [3], [9], [10]).

Since $\cap_{\nu} \Re_{v}=\{0\}$, it follows that the $w^{*}$-closed span $w^{*}-V_{\nu} \mathfrak{T}_{\nu}^{\perp}$ of the $\Re_{v}^{\perp}$ 's is the whole dual space $X^{*}$. We have shown that $\sigma\left(T^{*}\right) \backslash \cup_{\nu} \sigma\left(T_{v}^{*}\right)$ $=\sigma(T) \backslash \sigma^{\prime} .\left(T_{v}^{*}=T^{*} \mid \Re_{v}^{\perp}\right)$. Thus, if $\lambda \in \sigma(T) \backslash \sigma^{\prime}$ we can proceed as in the proof of (i) to show that $\left(T^{*}-\lambda\right) X^{*} \supset \Sigma_{\nu} \Re_{\nu}^{\perp}$; hence $\operatorname{ran}\left(T^{*}-\lambda\right)$ is $w^{*}$-dense in $X^{*}$. If $\operatorname{ran}\left(T^{*}-\lambda\right)$ is not closed, then $\lambda \in \sigma_{\mathrm{ap}}\left(T^{*}\right)$ because $\left(T^{*}-\lambda\right)$ cannot be bounded below. If $\operatorname{ran}\left(T^{*}-\lambda\right)$ is closed, then $\operatorname{ran}\left(T^{*}-\right.$ $\lambda)=[\operatorname{ker}(T-\lambda)]^{\perp}$ is $w^{*}$-closed and, therefore, $\operatorname{ran}\left(T^{*}-\lambda\right)=X^{*},\left(T^{*}-\lambda\right)$ is a semi-Fredholm operator of positive index and $\lambda \in \sigma_{p}\left(T^{*}\right) \subset \sigma_{\text {ap }}\left(T^{*}\right)$ (see [9, Chapter IV]). This proves $\left(\mathrm{i}^{*}\right)$.

(ii*) Let $\sigma_{0}$ be a clopen subset of $\sigma(T)$ which does not intersect $\sigma^{\prime}$. For fixed $\nu$, let $f_{\nu}$ be defined as in the proof of (ii), with $\sigma\left(T_{\nu}\right)$ replaced by $\sigma\left(\bar{T}_{v}\right)$. Let $E_{\nu}=f_{v}(T)$; then $E_{v}^{*}=\left[f_{\nu}(T)\right]^{*}=f_{\nu}\left(T^{*}\right)$ and $E=E_{v}, E^{*}=E_{\nu}^{*}$ are independent of $\nu$. Since $f_{v}$ is analytic in a neighborhood of $\sigma(T) \cup \sigma\left(\bar{T}_{v}\right)$ and $\bar{T}_{p}-\lambda \in \mathcal{L}\left(\mathcal{X} / \mathscr{T}_{p}\right)$ is the operator induced by $T-\lambda$ on $\mathcal{X} / \mathscr{N}_{p}$, it follows that $\Re_{v} \in$ Lat $E_{v}$ and $\bar{E}_{v}=f_{v}\left(\bar{T}_{\nu}\right)$ is equal to the operator induced by $E_{v}=E$ on $\mathcal{X} / \mathscr{T}_{\nu}$. Then $\mathcal{X}=\operatorname{ran} E \oplus \operatorname{ker} E$ and $X^{*}=\operatorname{ran} E^{*} \oplus \operatorname{ker} E^{*}$, $\operatorname{ker} E^{*}=(\operatorname{ran} E)^{\perp}$ and $\operatorname{ran} E^{*}=(\operatorname{ker} E)^{\perp}$. By using the canonical identification of $\left(\mathrm{i}^{*}\right)$, it is not difficult to see that $\left(\operatorname{ker} E_{\nu}\right)^{\perp} \supset\left(\operatorname{ker} \bar{E}_{\nu}\right)^{\perp}=\Re_{\nu}^{\perp}$. Hence, $\operatorname{ran} E^{*}$ is a $w^{*}$-closed subspace of $X^{*}$ containing $\Sigma_{\nu} \Re_{\nu}^{\perp}$, which is a $w^{*}$-dense linear manifold; therefore $\operatorname{ran} E^{*}=X^{*}$, whence we obtain that $E^{*}=I^{*}$ and $\sigma_{0}=\varnothing$.

The proof of (ii*) is complete. Finally, the proofs of (iii*) and (iv*) are identical to those of (iii) and (iv), resp.

2. Triangular operators. In this section we shall improve and extend several results of J. D. Stafney (see [11]) about the spectra of lower triangular matrices of a certain type. Let $\Re, \Re \in$ Lat $T$, such that $\Re \subset \Re$ and $\operatorname{dim} \pi / \Re=1$; then the eigenvalue of the one-dimensional operator induced by $T \mid \Re$ on $\Re / \Re$ will be called a diagonal entry of $T$.

TheOREM 2. Let $T \in \mathcal{L}(\mathfrak{X})$ and let $\mathcal{C}$ be a chain in Lat $T$ such that:

(1) $\mathcal{C}=\left\{\Re_{v}: \nu \in \Phi\right\}$ is well-ordered from below; i.e., $\Phi$ is an initial segment of the ordinals and $\alpha, \beta \in \Phi, \alpha \leqslant \beta$ implies that $\mathfrak{N}_{\alpha} \subset \mathfrak{T}_{\beta}$.

(2) $\mathfrak{N}_{0}=\{0\}$; for each $\nu \in \Phi$, dim $\Re_{\nu+1} / \Re_{\nu}=1$, and for each limit ordinal $\gamma, \Re_{\gamma}=\bigvee\left\{\Re_{\nu}: \nu<\gamma\right\}$.

(3) $\mathcal{X}=\bigvee\left\{\Re_{\nu}: \nu \in \Phi\right\}$.

Then: (i) $\mathcal{C} \subset$ Lat $\mathbb{Q}_{T}^{a} ;\left\{\sigma\left(T_{\nu}\right): \nu \in \Phi\right\}$ is an increasing family of compact subsets of $\sigma(T)$ and $\sigma(T)=\sigma_{\mathrm{ap}}(T)\left(T_{\nu}=T \mid \Re_{\nu}\right)$. 
(ii) Every clopen subset of $\sigma(T)$ intersects $d(T)=\left\{\lambda_{\nu}: \nu \in \Phi\right\}$, where $\lambda_{\nu}$ is the diagonal entry of $T$ corresponding to $\left[\Re_{\nu}, \Re_{\nu+1}\right]$, and every component of $\sigma(T)$ intersects $d(T)^{-}$, the closure of $d(T)$. In particular, every isolated point of $\sigma(T)$ is a diagonal entry; moreover, these isolated points are eigenvalues of $T$.

(iii) For each $\nu \in \Phi, \sigma(T)=\sigma\left(T_{\nu}\right) \cup \sigma\left(\bar{T}_{\nu}\right)$, where $\bar{T}_{\nu}$ is the operator induced by $T$ on $X / \Re_{\nu}$, and $\left\{\sigma\left(\bar{T}_{\nu}\right): \nu \in \Phi\right\}$ is a decreasing family of compact subsets of $\sigma(T)$.

(iv) Let $\bar{\sigma}_{f}(T)=\bigcap\left\{\sigma\left(\bar{T}_{\nu}\right): \nu \in \Phi\right\}$. If $\Phi=\{0,1,2, \ldots\}$, then $\sigma(T)=$ $\bar{\sigma}_{f}(T) \cup d(T)$.

Proof. (i) Let $\Phi=\{0,1,2, \ldots, \omega, \omega+1, \ldots, \nu, \nu+1, \ldots\}$. For each finite $n, \mathscr{T}_{n} \in$ Lat $\mathbb{Q}_{T}^{a}$ and $\sigma\left(T_{n}\right)=\left\{\lambda_{0}, \lambda_{1}, \ldots, \lambda_{n-1}\right\}$ (or $\varnothing$, if $n=0$; see [5, Theorem 8]) and $\left\{\sigma\left(T_{n}\right): n=0,1,2, \ldots\right\}$ is an increasing sequence of finite subsets of $\sigma(T)$.

Now we proceed by transfinite induction: if $\mathscr{T}_{v} \in$ Lat $\mathbb{Q}_{T}^{a}$ and $\sigma\left(T_{v}\right)=$ $\sigma_{\text {ap }}\left(T_{v}\right)$, then $\Re_{\nu} \in$ Lat $T_{p+1}$ and (since $\operatorname{dim} \mathfrak{R}_{\nu+1} / \mathscr{N}_{\nu}=1$ ) it follows from [5, Theorem 8] that $\mathfrak{T}_{\nu} \in$ Lat $\mathbb{Q}_{T_{r+1}}^{a}$, which is contained in Lat $\mathbb{Q}_{T}^{a}[6$, Theorem 6.1]; moreover, $\sigma\left(T_{\nu+1}\right)=\sigma\left(T_{\nu}\right) \cup\left\{\lambda_{\nu}\right\}=\sigma_{\text {ap }}\left(T_{\nu+}\right) \cup\left\{\lambda_{\nu}\right\}=$ $\sigma_{\mathrm{ap}}\left(T_{\nu+1}\right)$, because $\sigma\left(T_{\nu+1}\right) \backslash \sigma_{\mathrm{ap}}\left(T_{\nu+1}\right)$ is an open subset of $\mathrm{C}$ contained in $\left\{\lambda_{\nu}\right\}$ (in fact, $\left[\sigma\left(T_{\nu+1}\right) \backslash \sigma_{\mathrm{ap}}\left(T_{\nu+1}\right)\right] \cap \sigma_{\mathrm{ap}}\left(T_{\nu}\right)=\varnothing$ ) and therefore it must be empty. If $\gamma$ is a limit ordinal and $\mathfrak{R}_{\nu} \in$ Lat $\mathbb{Q}_{T}^{a}$ and $\sigma\left(T_{\nu}\right)=\sigma_{\text {ap }}\left(T_{\nu}\right)$ for all $\nu<\gamma$, then $\mathscr{N}_{\gamma} \in$ Lat $\mathbb{Q}_{T}^{a}$ because $\mathscr{R}_{\gamma}=\bigvee\left\{\mathscr{N}_{\nu}: \nu<\gamma\right\}$ and Lat $\mathbb{Q}_{T}^{a}$ is complete; moreover, by Theorem 1(i),

$$
\sigma\left(T_{\gamma}\right)=\sigma_{\text {ap }}\left(T_{\gamma}\right) \cup\left\{\bigcup_{\nu<\gamma} \sigma_{\text {ap }}\left(T_{\nu}\right)\right\}=\sigma_{\text {ap }}\left(T_{\gamma}\right),
$$

which is clearly contained in $\sigma(T)$.

We have shown, in particular, that $\left\{\sigma\left(T_{\nu}\right)\right\}$ is an increasing family of sets and that $\mathcal{C} \in$ Lat $\mathbb{Q}_{T}^{a}$. Finally, by applying Theorem 1(i) to $T$ and using (3), we conclude that $\sigma(T)=\sigma_{\text {ap }}(T)$.

(ii) Let $\sigma_{0}$ be a nonempty clopen subset of $\sigma(T)$ and let $E_{0}$ be the associated idempotent. Then $E_{0} \in \mathbb{Q}_{T}^{a}$ and $\mathcal{X}=\operatorname{ran} E_{0} \oplus \operatorname{ker} E_{0}$, where ran $E_{0}$, ker $E_{0}$ $\in$ Lat $\mathbb{Q}_{T}^{a}, \sigma\left(T \mid \operatorname{ran} E_{0}\right)=\sigma_{0}$ and $\sigma\left(T \mid \operatorname{ker} E_{0}\right) \cap \sigma_{0}=\varnothing$. $\mathbb{Q}_{T}^{a}$ and Lat $\mathbb{Q}_{T}^{a}$ split with respect to the above decomposition of $\mathcal{X}$ and, therefore (since $\mathcal{C} \subset$ Lat $\left.\mathbb{Q}_{T}^{a}\right), \mathfrak{K}_{\nu}=\left(\mathfrak{R}_{\nu} \cap \operatorname{ran} E_{0}\right) \oplus\left(\mathfrak{R}_{\nu} \cap \operatorname{ker} E_{0}\right)$ for all $\nu \in \Phi$ (see [6]).

Condition (3) guarantees that $E_{0} \Re_{\nu}=\Re_{\nu} \cap \operatorname{ran} E_{0} \neq\{0\}$ for all $\nu$ in a final segment of $\Phi$. Let $\gamma$ be the first index such that $E_{0} \mathfrak{R}_{\gamma} \neq\{0\}$. Since $\sigma\left(T_{\gamma}\right) \subset \sigma(T), \sigma_{0} \cap \sigma\left(T_{\gamma}\right)$ is a nonempty clopen subset of $\sigma\left(T_{\gamma}\right)$ and therefore, by Theorem 1(ii), $\sigma_{0} \cap \sigma\left(T_{\gamma}\right)$ must intersect $U\left\{\sigma\left(T_{\nu}\right): \nu<\gamma\right\}$; but this implies that $\sigma_{0}$ intersects $\sigma\left(T_{\nu}\right)$ for some $\nu<\gamma$, contradicting the definition of $\gamma$, unless $\gamma=\alpha+1$ for some ordinal $\alpha \in \Phi$ such that $\sigma_{0} \cap \sigma\left(T_{\alpha}\right)=\varnothing$. It follows that $\sigma_{0} \cap \sigma\left(T_{\gamma}\right)=\sigma_{0} \cap \sigma\left(T_{\alpha+1}\right)=\left\{\lambda_{\alpha}\right\}$, i.e. $\lambda_{\alpha} \in \sigma_{0}$. 
This proves the first part of (ii); the second statement follows from the first one as in the proof of Theorem 1 (iii). The fact that every isolated point of $\sigma(T)$ belongs to $d(T)$ is also clear; thus, it only remains to show that the isolated points are eigenvalues of $T$. Let $E_{\gamma}$ be the idempotent associated to $\left\{\lambda_{\gamma}\right\}$, where $\lambda_{\gamma} \in d(T)$ is an isolated point of $\sigma(T)$. Without loss of generality we can assume that $\gamma$ is the first index such that $\lambda_{\nu}=\lambda_{\gamma}$. Then $\Re_{\gamma+1}=\Re_{\gamma}$ $\oplus\left\{c x_{\gamma}: c \in \mathbf{C}\right\}$, where $\Re_{\gamma}=\Re_{\gamma+1} \cap \operatorname{ker} E_{\gamma}, \sigma\left(T_{\gamma}\right)$ does not contain the point $\lambda_{\gamma}$ and $x_{\gamma}$ is any nonzero vector of the one-dimensional subspace $\Re_{\gamma+1} \cap \operatorname{ran} E_{\gamma}$. It is easy to see that $T_{\gamma+1} x_{\gamma}=\lambda_{\gamma} x_{\gamma}$ and, therefore, $\lambda_{\gamma} \in$ $\sigma_{p}\left(T_{\gamma+1}\right) \subset \sigma_{p}(T)$.

(iii) The equality $\sigma(T)=\sigma\left(T_{\nu}\right) \cup \sigma\left(\bar{T}_{\nu}\right)$ follows from (i) and [5, Theorem 3]. As in the proof of (i), we can easily see that $\sigma\left(\bar{T}_{\nu}\right)=\sigma\left(\bar{T}_{\nu+1}\right) \cup\left\{\lambda_{\nu}\right\}$ $\left(\lambda_{\nu} \in \sigma_{p}\left(\bar{T}_{\nu}\right)\right)$; hence, $\sigma\left(\bar{T}_{\nu}\right) \supset \sigma\left(\bar{T}_{\nu+1}\right)$ for all $\nu \in \Phi$. In general, if $\alpha, \beta \in \Phi$ and $\alpha<\beta$, then $X / \mathscr{N}_{\beta}$ is canonically isomorphic to $\left(\mathscr{X} / \mathscr{T}_{\alpha}\right) /\left(\mathscr{T}_{\beta} / \mathscr{T}_{\alpha}\right)$ and, according to [5, Theorem 3], $\sigma\left(\bar{T}_{\alpha}\right)=\sigma\left(\bar{T}_{\beta}\right) \cup \sigma\left(\bar{T}_{\alpha} \mid \mathscr{T}_{\beta} / \mathscr{T}_{\alpha}\right) \supset \sigma\left(\bar{T}_{\beta}\right)$, provided $\mathfrak{T}_{\beta} / \mathscr{T}_{\alpha} \in$ Lat $\mathbb{Q}_{\bar{T}_{\alpha}}^{a}$; but this is a consequence of (i). Indeed, it is enough to replace (in the proof of (i)) $T$ by $\bar{T}_{\alpha}$ and $\mathcal{C}$ by $\left\{\Re_{\nu} / \Re_{\alpha}: \nu \in \Phi\right.$, $\nu \geqslant \alpha\}$. Hence, $\sigma\left(\bar{T}_{\alpha}\right)$ always contains $\sigma\left(\bar{T}_{\beta}\right)$ whenever $\alpha, \beta \in \Phi$ and $\alpha<\beta$. Therefore,

$$
\begin{array}{r}
\sigma(T) \supset \sigma\left(\bar{T}_{1}\right) \supset \sigma\left(\bar{T}_{2}\right) \supset \cdots \supset \sigma\left(\bar{T}_{\nu}\right) \supset \sigma\left(\bar{T}_{\nu+1}\right) \\
\supset \cdots \supset \bar{\sigma}_{f}(T)=\bigcap_{\nu} \sigma\left(\bar{T}_{\nu}\right)
\end{array}
$$

$(\neq \varnothing$, unless $\mathfrak{X} \in \mathcal{C})$.

(iv) Let $\Phi=\{0,1,2, \ldots\}$. The proofs of (i) and (iii) show that $\sigma(T)=$ $d(T) \cup \bar{\sigma}_{f}(T)$.

Roughly speaking, the operator $T$ of Theorem 2 has an "upper triangular matrix" with respect to the chain $\mathcal{C}$. Analogous results can be proven for an operator having a "lower triangular matrix" with respect to a certain chain of invariant subspaces, by using the fact [5, Theorem 8] that an invariant subspace of finite codimension always belongs to Lat $\mathbb{Q}_{T}^{a}$, and Theorem $1^{*}$ instead of Theorem 1. Thus, we shall establish here the "dual version" of Theorem 2. The proof is left to the interested reader (if any!).

THEOREM 2*. Let $T \in \mathcal{E}(\mathcal{X})$ and let $\mathcal{C}$ be a chain in Lat $T$ such that:

(1) $\mathcal{C}=\left\{\Re_{\nu}: \nu \in \Phi\right\}$ is well-ordered from above; i.e., $\Phi$ is an initial segment of the ordinals and $\alpha, \beta \in \Phi, \alpha<\beta$ implies that $\Re_{\alpha} \supset \Re_{\beta}$.

(2) $\mathfrak{\pi}_{0}=X$; for each $\nu \in \Phi, \operatorname{dim} \Re_{\nu} / \Re_{\nu+1}=1$ and for each limit ordinal $\gamma, \mathscr{R}_{\gamma}=\bigcap\left\{\mathfrak{N}_{\nu}: \nu<\gamma\right\}$.

(3) $\{0\}=\cap\left\{\mathscr{N}_{v}: \nu \in \Phi\right\}$.

Then: $\left(\mathrm{i}^{*}\right) \mathcal{C} \subset$ Lat $\mathbb{Q}_{T}^{a} ;\left\{\sigma\left(\bar{T}_{\nu}\right): \nu \in \Phi\right\}$ is an increasing family of compact 
subsets of $\sigma(T)$ and $\sigma\left(T^{*}\right)=\sigma_{\mathrm{ap}}\left(T^{*}\right)\left(T_{\nu}\right.$ and $\bar{T}_{\nu}$ are defined exactly as in Theorem 2).

(ii*) Every clopen subset of $\sigma(T)$ intersects $d(T)$ and every component of $\sigma(T)$ intersects $d(T)^{-}$. In particular, every isolated point of $\sigma(T)$ is a diagonal entry; moreover, these isolated points are eigenvalues of $T^{*}$.

(iii*) For each $\nu \in \Phi, \sigma(T)=\sigma\left(\bar{T}_{\nu}\right) \cup \sigma\left(T_{\nu}\right)$ and $\left\{\sigma\left(T_{\nu}\right): \nu \in \Phi\right\}$ is a decreasing family of compact subsets of $\sigma(T)$.

(iv*) Let $\sigma_{f}(T)=\cap\left\{\sigma\left(T_{v}\right): \nu \in \Phi\right\}$. If $\Phi=\{0,1,2, \ldots\}$, then $\sigma(T)=$ $\sigma_{f}(T) \cup d(T)$.

None of the inclusions $\sigma_{p}(T) \subset d(T)$ and $d(T) \subset \sigma_{p}(T)$ is true in general. Indeed, we have the following counterexamples:

EXAMPLE F. Let $S$ be the unilateral shift "multiplication by $e^{i x}$ " in the Hardy space $H^{2}(D)=\bigvee\left\{e^{i n x}\right\}_{n=0}^{\infty}$ (the $e^{i n x}$ 's form an orthonormal basis of this Hilbert space). Then $\left\{\Re_{n}=\bigvee\left(e^{i k y}\right)_{k=0}^{n}: n=0,1, \ldots\right\}$ is an invariant subspace chain for $S^{*}$ satisfying the conditions (1), (2), (3) and (iv) of Theorem 2 and $d\left(S^{*}\right)=\{0\}=d\left(S^{*}\right)^{-}$. On the other hand, $\sigma_{p}\left(S^{*}\right)=D$ (see [3], [4]).

EXAMPLE G. Let $T_{0} \in \mathcal{L}\left(\mathscr{X}_{0}\right)$ be the operator defined in Example $\mathrm{E}$ and let $\mathfrak{X}=\mathfrak{X}_{0} \oplus \mathrm{C}$. The structure of $T_{0}$ makes it clear that $T_{0}$ has an invariant subspace chain $C_{0}$ satisfying (1), (2), (3) and (iv) of Theorem 2. Theorem 1(i) and the fact that $T_{0}$ is unitarily equivalent to its adjoint implies (after some computations, see [3], [8]) that $\sigma_{p}\left(T_{0}\right)=\sigma_{p}\left(T_{0}^{*}\right)=\{0\}$ and $\sigma_{\text {ap }}\left(T_{0}\right)=\sigma_{\text {ap }}\left(T_{0}^{*}\right)$ $=D^{-}$. Therefore, for every $\lambda \in D^{-} \backslash\{0\}, \operatorname{ran}\left(T_{0}-\lambda\right)$ is a proper dense linear manifold of $\mathscr{X}_{0}$ and $\operatorname{ker}\left(T_{0}-\lambda\right)=\{0\}$. Fix $\lambda$ and choose $x_{\lambda} \in \mathscr{X}_{0} \backslash$ $\operatorname{ran}\left(T_{0}-\lambda\right)$; then define $T \in \mathcal{L}(\mathscr{X})$ by means of the matrix

$$
T=\left(\begin{array}{ll}
T_{0} & A_{\lambda} \\
0 & \lambda
\end{array}\right)
$$

(acting in the usual fashion on $\mathcal{X}=\mathscr{X}_{0} \oplus \mathrm{C}$ ) where $A_{\lambda}(1)=x_{\lambda}$. Then $\mathcal{C}=$ $\mathcal{C}_{0} \cup\left\{\mathfrak{X}_{0} \oplus(0), \mathfrak{X}\right\}$ is a chain of invariant subspaces of $T$ satisfying (1), (2) and (3) (the type of order being equal to $\omega+2)$ and $d(T)=\{0, \lambda\}$. However, a straightforward computation shows that $\operatorname{ker}(T-\lambda)=\{0\}$. Indeed, $\sigma_{p}(T)$ $=\{0\}$.

Let $\left\{\lambda_{\alpha}: \alpha \in \Xi\right\}$ be an arbitrary subset of $D^{-}$, well-ordered according to the index set $\Xi$. For each $\alpha \in \Xi$, define $T_{\alpha}$ as the operator $T$ given above, where $x_{\alpha} \in \mathscr{X}_{0} \backslash \operatorname{ran}\left(T_{0}-\lambda_{\alpha}\right)$ is chosen to be a norm-one vector (hence, $\left\|T_{\alpha}\right\|$ is a constant independent of $\alpha$ ), acting on the Hilbert space $X_{\alpha}$ (a copy of the above $\mathcal{X}$ ), and let $\mathcal{C}_{\alpha}$ be the copy of the above $\mathcal{C}$. Finally, define $\mathcal{Y}$ to be the orthogonal direct sum of the $\mathscr{X}_{\alpha}$ 's and $L=\bigoplus_{\alpha} T_{\alpha}$; clearly, $\|L\|=\left\|T_{\alpha}\right\|<$ $\infty$ and, therefore, $L \in \mathcal{L}(Y)$, and it is easy to see that $\mathscr{D}=\times_{\alpha} e_{\alpha}$ (lexicographically ordered) is a chain for $L$ satisfying (1), (2) and (3) of 
Theorem 1, $d(D)=\left\{\lambda_{\alpha}: \alpha \in \Xi\right\} \cup\{0\}$ and we still have $\sigma_{p}(L)=\{0\}$ !

The results of Theorems 2 and $2^{*}$ can be only partially extended to other lattices. Let $\Lambda$ be a subset of $\mathbf{C}$ containing at most one point of each bounded component of $\rho(T)$ and let $\mathbb{Q}_{T}(\Lambda)$ denote the weakly closed algebra generated by $T$ and $\left\{(T-\lambda)^{-1}: \lambda \in \Lambda\right\}$. If $\Omega=\rho_{\infty}(T) \cup\left[\cup\left\{\rho_{\lambda}(T): \lambda \in \Lambda\right\}\right]$, where $\rho_{\lambda}(T)$ is the component of $\rho(T)$ containing $\lambda$, then we shall write $\sigma_{\Lambda}(T)=$ $\hat{\sigma}(T) \backslash \Omega$. Then, as a corollary of Theorem 7 of [5], we have

Corollary 3. Let $\mathcal{C}=\left\{\mathscr{T}_{\nu}: \nu \in \Phi\right\}$ (where the index set $\Phi$ is totally ordered from below by inclusion of the corresponding subspaces) be a chain in Lat $\mathbb{Q}_{\underline{T}}(\Lambda)$. Then $\sigma_{\Lambda}(T)=\sigma_{\Lambda}\left(T_{\nu}\right) \cup \sigma_{\Lambda}\left(\bar{T}_{\nu}\right)$ for all $\nu \in \Phi$ and $\left\{\sigma_{\Lambda}\left(T_{\nu}\right): \nu \in \Phi\right\}$ $\left(\left\{\sigma_{\Lambda}\left(\bar{T}_{\nu}\right): \nu \in \Phi\right\}\right.$, resp.) is an increasing (decreasing, resp.) family of compact subsets of $\sigma_{\Lambda}(T)$.

This corollary cannot be improved in general, because the "holes" of $\sigma\left(T_{\nu}\right)$ can suddenly disappear if $\nu$ is a limit point of the index set. Namely, we have

EXAMPLE $H$. Let $T$ be the bilateral shift in $L^{2}(\partial D, d m)$, as in [5, Example A] (see also [3], [4]) and let $L=(T+2) \oplus(T-2) \in \mathcal{L}\left(L^{2} \oplus L^{2}\right)$. Define $\mathcal{C}$ as follows: if $\mathscr{R}_{n}=\bigvee\left\{e^{i k x}\right\}_{k=n}^{\infty}, n=0, \pm 1, \pm 2, \ldots$, then $\mathcal{C}=\left\{\mathscr{T}_{n} \oplus(0)\right.$ $\left.=\Re_{n}^{-}\right\} \cup\left\{L^{2} \oplus(0)\right\} \cup\left\{L^{2} \oplus \mathbb{N}_{n}^{+}\right\}$. It is easy to see that $C$ is a chain of invariant subspaces of $L, L^{2} \oplus L^{2}=\bigvee\{\Re \in \mathcal{C}\},\{0\}=\cap\{\Re \in \mathcal{C}\}$, $\sigma\left(L \mid \pi_{n}\right)$ is a closed disc of radius one centered at $(-2)$ and $\sigma\left(L \mid \pi_{n}^{+}\right)$is the union of $\partial \sigma\left(L \mid \Re_{n}^{-}\right)$with a closed disc of radius one centered at 2 , while $\sigma\left(L \mid L^{2} \oplus\{0\}\right)$ is just the circle of radius one and center $(-2)$. This example also shows that, in general, a component of $\sigma(L)$ need not contain a diagonal entry.

Lemma 4. Let $T \in \mathfrak{L}(\mathcal{X})$ and let $\mathcal{C} \subset$ Lat $\mathbb{Q}_{T}(\Lambda)$ be a maximal chain of subspaces of $X$. Let $\sigma_{0}$ be a nonempty clopen subset of $\sigma_{\Lambda}(T)$ with associated idempotent $E_{0}$. If $T_{0}$ denotes the restriction of $T$ to $\operatorname{ran} E_{0}$, then $C_{0}=\left\{T_{v} \cap\right.$ $\left.\operatorname{ran} E_{0}: \nu \in \Phi\right\}$ is a maximal chain of subspaces of $\operatorname{ran} E_{0}$ contained in Lat $\mathbb{Q}_{T_{0}}(\Lambda)$ and every diagonal entry of $T_{0}$ (with respect to the chain $\mathcal{C}_{0}$ ) is also a diagonal entry of $T$.

Proof. A maximal chain of subspaces can be characterized by the following two properties: $\mathcal{C}$ is complete and, whenever $\Re, \Re \in \mathcal{C}, \Re \subset \Re$ and there is no $\mathbb{N}^{\prime} \in \mathcal{C}$ such that $\mathscr{N} \subsetneq \mathfrak{N}^{\prime} \subsetneq \Re$, then $\operatorname{dim} \Re / \mathscr{N}=1$. On the other hand, it is clear that $E_{0} \in \mathbb{Q}_{T}^{\mp}(\Lambda)$ and, therefore, Lat $\mathbb{Q}_{T}(\Lambda)$ splits with respect to the decomposition $\mathcal{X}=\operatorname{ker} E_{0} \oplus \operatorname{ran} E_{0}$ [5], [6]. It follows that $\bigodot_{0}$ is a maximal chain of subspaces of $\operatorname{ran} E_{0}$ contained in Lat $\mathbb{Q}_{T_{0}}(\Lambda)$.

Let $\Re, \pi \in \mathcal{C}_{0}$ be a pair of subspaces such that $\Re \subsetneq \Re$ and $\operatorname{dim} \pi / \Re=1$, and let $\lambda$ be the corresponding diagonal entry. Define

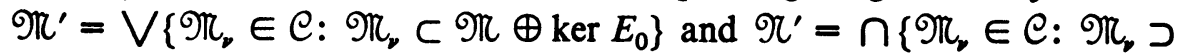


$\left.\Re \oplus \operatorname{ker} E_{0}\right\}$. Clearly, $\Re^{\prime}, \Re^{\prime} \in \mathcal{C}$ and $\Re^{\prime} \subsetneq \Re^{\prime}$; moreover, the maximality of $\mathcal{C}$ implies that $\operatorname{dim} \Re^{\prime} / \Re^{\prime}=1$. Therefore, there exists $\Re^{\prime \prime} \subset$ ker $E_{0}$ such that $\Re^{\prime}=\mathscr{T} \oplus \Re^{\prime \prime}$ and $\Re^{\prime}=\Re \oplus \Re{ }^{\prime \prime}$ (to see this, use the fact that $\mathcal{C}$ splits), whence it readily follows that the diagonal entry of $T$ corresponding to the pair $\Re^{\prime}, \Re^{\prime}$ is the above $\lambda$.

Corollary 5. Let $T \in \mathcal{L}(\mathcal{X})$ and let $\mathcal{C} \subset$ Lat $\mathcal{Q}_{T}(\Lambda)$ be a chain such that:

(1) $\mathcal{C}$ is a maximal chain of subspaces of $\mathcal{X}$.

(2) Given $\Re \in \mathcal{C}$, there exists $\Re \in \mathcal{C}, \Re \neq \Re$, such that either $\Re \subset$ $\Re$ and $\left\{\Re^{\prime} \in \mathcal{C}: \mathfrak{N} \subset \mathfrak{N}^{\prime} \subset \Re\right.$ \} is a well-ordered (from above or from below!) "segment" of $\mathcal{C}$, or $\Re \subset \Re$ and $\{\Re$ ' $\in \mathcal{C}: \Re \subset \Re$ ' $\subset \Re$ \} is a well-ordered (from above or from below!) "segment" of $\mathcal{C}$.

Then every clopen subset of $\sigma_{\Lambda}(T)$ contains a diagonal entry, every component of $\sigma_{\Lambda}(T)$ intersects $d(T)^{-}$and every isolated point of $\sigma_{\Lambda}(T)$ is a diagonal entry.

Proof. As in the proofs of Theorems 2 and $2^{*}$, it will be enough to show that every clopen subset of $\sigma_{\Lambda}(T)$ contains a diagonal entry. Let $\sigma_{0}$ be a nonempty clopen subset of $\sigma_{\Lambda}(T)$. Now observe that Lemma 4 reduces our problem to show that $\sigma_{0}$ contains a diagonal entry of the restriction of $T$ to $\operatorname{ran} E_{0}$, where $E_{0}$ is the idempotent associated to $\sigma_{0}$. In other words: it is enough to prove the result for the case when $\sigma_{0}=\sigma_{\Lambda}(T)$.

Let $\Re, \pi$ be any pair of subspaces in $\mathcal{C}$ such that $\Re \subsetneq \Re$ and the segment $[\Re, \Re]$ is well-ordered, and consider the operator $L \stackrel{\neq}{=}$ the operator induced by $T \mid \mathcal{R}$ on $\Re / \Re$. Then (see [5]) $\sigma_{\Lambda}(L) \subset \sigma_{\Lambda}(T)$ and Theorems 2 and $2^{*}$ guarantee that $\sigma_{\Lambda}(L) \cap d(L) \neq \varnothing$. Since $d(L) \subset d(T)$, the proof is complete.

\section{The case when the restrictions have disjoint spectra.}

THEOREM 6. Let $\mathfrak{T}_{1}, \ldots, \mathfrak{T}_{n}$ be a finite family of subspaces invariant under the operator $T$ and assume that $\sigma\left(T \mid \Re_{k}\right)$ has empty intersection with $\cup_{j \neq k} \sigma\left(T \mid \Re_{j}\right)$ for each $k=1, \ldots, n$. Then the algebraic sum of the $\mathfrak{M}_{j}^{\prime} s$ is direct and, moreover, the direct sum $\bigoplus_{j=1}^{n} \Re_{j}$ is closed in $\mathcal{X}$. In addition, if $\bigoplus_{j=1}^{n} \Re_{j}=\mathscr{X}$, then the $\mathfrak{K}_{j}$ 's are actually hyperinvariant subspaces of $T$ and $\sigma(T)=\bigcup_{j=1}^{n} \sigma\left(T \mid \mathscr{T}_{j}\right)$.

We shall need an auxiliary result. Indeed, the following lemma proves more than what we need for the proof of Theorem 6.

LEMMA 7. Let $\Re, \Re$ be two invariant subspaces of $T$ and assume that $\sigma(T \mid \Re) \cap \sigma(T \mid \Re)=\varnothing$. Assume, moreover, that $\sigma(T \mid \Re)=\bigcup_{j=1}^{k} \sigma_{2 j-1}$ and $\sigma(T \mid \mathcal{R})=\cup_{j=1}^{k} \sigma_{2 j}$, where the $\sigma_{2 j-1}$ 's $\left(\sigma_{2 j}\right.$ 's, resp.) are clopen subsets of $\sigma(T \mid \Re)(\sigma(T \mid \mathcal{T})$, resp.) and that there exist a polynomial $p(z)$ and real constants $\delta_{0}<0<\delta_{1}<\cdots<\delta_{2 k}$ such that $\sigma_{h} \subset\left\{z: \delta_{h-1}<|p(z)|<\delta_{h}\right\}$, 
$h=1,2, \ldots, 2 k$. Then $\Re \cap \Re=\{0\}$ and $\Re \oplus \Re$ (algebraic direct sum) is closed in $\mathcal{X}$.

Proof. Let $\mathscr{X}_{0}=\Re \cap \Re$; clearly, $\mathscr{X}_{0}$ is invariant under $T$. Thus, according to [1], $\partial \sigma\left(T \mid \mathscr{X}_{0}\right) \subset \sigma(T \mid \Re) \cap \sigma(T \mid \Re)=\varnothing$; hence, $\sigma\left(T \mid \mathscr{X}_{0}\right)=$ $\varnothing$ and, therefore, $\mathscr{X}_{0}=\{0\}$.

By the Riesz functional calculus $[10$, p. 421], $\Re$ can be written as the algebraic direct sum $\Re=\bigoplus_{j=1}^{\mathrm{k}} \Re_{2 j-1}$, where $\Re_{2 j-1}$ is invariant under $T$ and $\sigma\left(T \mid \Re_{2 j-1}\right)=\sigma_{2 j-1}, j=1, \ldots, k$. Similarly we have $\Re=\bigoplus_{j=1}^{k} \Re_{2 j}$, where $\Re_{2 j}$ is invariant under $T$ and $\sigma\left(T \mid \Re_{2 j}\right)=\sigma_{2 j}, j=1, \ldots, k$.

Let us assume that $\Re \oplus \Re$ is not closed in $X$; then (see [9, p. 219]) there exist two sequences of vectors $\left\{x_{n}\right\}_{n=1}^{\infty},\left\{y_{n}\right\}_{n=1}^{\infty}, x_{n} \in \Re, y_{n} \in \Re,\left\|x_{n}\right\|=$ $\left\|y_{n}\right\|=1$, for all $n$, such that $\lim (n \rightarrow \infty)\left\|x_{n}-y_{n}\right\|=0$. By using the above decompositions, we can write $x_{n}=\sum_{j=1}^{k} x_{n}^{2 j-1}, y_{n}=\sum_{j=1}^{k} y_{n}^{2 j}$, where $x_{n}^{2 j-1} \in$ $\mathscr{T}_{2 j-1}$ and $y_{n}^{2 j} \in \mathscr{T}_{2 j}, j=1, \ldots, k$. Moreover, since $\Re=\left(\bigoplus_{j=1}^{k-1} \mathscr{T}_{2 j}\right) \oplus$ $\Re_{2 k}$, the projection $P_{2 k}$ of $\Re$ onto $\Re_{2 k}$ along $\bigoplus_{j=1}^{k-1} \Re_{2 j}$ is bounded in $\Re$ and therefore there exists a positive constant $C_{2 k}$ such that $\left\|y_{n}^{2 k}\right\|=\left\|P_{2 k} y_{n}\right\|$ $<C_{2 k}$, for all $n$.

Claim. If $\left\|x_{n}-y_{n}\right\| \rightarrow 0$, then $\left\|y_{n}^{2 k}\right\| \rightarrow 0(n \rightarrow \infty)$. Assume it is not true; then, passing if necessary to a subsequence, we can assume that $\left\|y_{n}^{2 k}\right\|>\varepsilon>$ 0 , for some $\varepsilon$ and for all $n$.

Let $A=\left(1 / \delta_{2 k-1}\right) p(T)$. Clearly, every invariant subspace of $T$ is also invariant under $A$ and, by the spectral mapping theorem (see [10, p. 432]),

$$
\sigma(A \mid \Re)=\left(1 / \delta_{2 k-1}\right) p[\sigma(T \mid \Re)] \subset\{z:|z|<r<1\},
$$

for some $r, 0<r<1$,

$$
\begin{aligned}
\sigma\left(A \mid \oplus_{j=1}^{k-1} \Re_{2 j}\right) & =\left(1 / \delta_{2 k-1}\right) p\left[\sigma\left(T \mid \bigoplus_{j=1}^{k-1} \Re_{2 j}\right)\right] \\
& \subset\left\{z:|z|<r^{\prime}<r<1\right\},
\end{aligned}
$$

for some $r^{\prime}, 0<r^{\prime}<r$, and

$$
\begin{aligned}
\sigma\left(A \mid \Im_{2 k}\right) & =\left(1 / \delta_{2 k-1}\right) p\left[\sigma\left(T \mid \Re_{2 k}\right)\right] \\
& =\left(1 / \delta_{2 k-1}\right) p\left(\sigma_{2 k}\right) \subset\{z:|z|>R\},
\end{aligned}
$$

for some $R>1$.

By using the properties of the spectral radius [10, p. 425], we conclude that, for all $m$ large enough,

$$
\left\|A^{m} y_{n}^{2 k}\right\| \geqslant R^{m}\left\|y_{n}^{2 k}\right\| \geqslant \varepsilon R^{m} \rightarrow \infty \quad(\text { as } m \rightarrow \infty),
$$

while

$$
\begin{aligned}
\left\|A^{m}\left(y_{n}-y_{n}^{2 k}-x_{n}\right)\right\| & \leqslant\left\|A^{m}\left(y_{n}-y_{n}^{2 k}\right)\right\|+\left\|A^{m} x_{n}\right\| \\
& \leqslant\left(1+C_{2 k}\right) r^{\prime m}+r^{m} \\
& \leqslant\left(2+C_{2 k}\right) r^{m} \rightarrow 0, \quad \text { as } m \rightarrow \infty .
\end{aligned}
$$


Since the above estimations do not depend on $n$, we can fix an $m_{0}$ so that $\left\|A^{m_{0}}\left(y_{n}-x_{n}\right)\right\|>1$ for all $n$; from $\left\|x_{n}-y_{n}\right\| \rightarrow 0(n \rightarrow \infty)$, it follows that

$$
1<\left\|A^{m_{0}}\left(x_{n}-y_{n}\right)\right\|<\left\|A^{m_{0}}\right\| \cdot\left\|x_{n}-y_{n}\right\| \rightarrow 0 \quad(n \rightarrow \infty),
$$

a contradiction. This proves our claim.

Hence, if $\left\|x_{n}-y_{n}\right\| \rightarrow 0$, then $y_{n}^{2 k} \rightarrow 0$ and, for all $n$ large enough, $\left\|y_{n}^{2 k}\right\|<\frac{1}{2}$; writing $y_{n}^{\prime}$ for $\left(1 /\left\|y_{n}-y_{n}^{2 k}\right\|\right)\left(y_{n}-y_{n}^{2 k}\right)$, it follows that $\| x_{n}-$ $y_{n}^{\prime} \| \rightarrow 0$ as $n \rightarrow \infty$. By repeating the same arguments, it follows that $x_{n}^{2 k-1} \rightarrow$ 0 ; then, by induction on $k$, we conclude that $x_{n} \rightarrow 0$ and $y_{n} \rightarrow 0$, a contradiction. Therefore $\Re \oplus \Re$ is closed in $\mathfrak{X}$.

ProOF OF THEOREM 6. Our hypothesis on the spectra of the operators $T \mid \Re_{j}, j=1, \ldots, n$, is equivalent to saying that there exists a finite family $\Gamma=\left\{\gamma_{1}, \ldots, \gamma_{m}\right\}$ of rectifiable pairwise disjoint closed Jordan curves such that $\sigma\left(T \mid \Re_{k}\right)$ is separated from $U_{j \neq k} \sigma\left(T \mid \Re_{j}\right)$ by some subset of the $\gamma_{h}$ 's. Clearly, we can assume that no subset of $m-1$ curves has the desired property (in other words, that $\Gamma$ is "minimal"); then, after a suitable renumbering of the curves, we can also assume that the interior of $\gamma_{1}$ (i.e., the bounded component of $\left.\mathbf{C} \backslash \gamma_{1}\right)$ does not contain any of the curves $\gamma_{2}, \ldots, \gamma_{m}$ and, by induction, that the interior of $\gamma_{k}$ does not contain any of the curves $\gamma_{k+1}, \ldots, \gamma_{m}$, for all $k=1,2, \ldots, m-1$.

Since $\Gamma$ is minimal, (int $\left.\gamma_{1}\right) \cap \sigma$ (where $\sigma=\cup_{j=1}^{n} \sigma\left(T \mid \Re_{j}\right)$ ) is a nonempty clopen subset of one of the $\sigma\left(T \mid \mathscr{T}_{j}\right)$ 's. Let us assume that (int $\left.\gamma_{1}\right) \cap \sigma=\sigma_{1}$ is a nonempty clopen subset of $\sigma\left(T \mid T_{1}\right)$; then the Riesz functional calculus gives a decomposition of the form $\Re_{1}=\Re_{1} \oplus \Re_{1}^{\prime}$, where $\Re_{1}$, $\Re_{1}^{\prime}$ are two invariant subspaces of $T$ such that $\sigma\left(T \mid \mathscr{T}_{1}\right)=\sigma_{1}, \sigma\left(T \mid \mathscr{T l}_{1}^{\prime}\right)$ is the (possibly empty) subset $\sigma\left(T \mid \mathscr{R}_{1}\right) \backslash \sigma_{1}$ and the spectra $\sigma\left(T \mid \mathscr{R}_{1}^{\prime}\right)$, $\sigma\left(T \mid \Re_{2}\right), \ldots, \sigma\left(T \mid \Re_{n}\right)$ are separated by the $m-1$ curves $\gamma_{2}, \ldots, \gamma_{m}$. By induction, we finally obtain a family of invariant subspaces $\Re_{1}, \ldots, \Re_{m}$, $\Upsilon_{m+1}$ with nonempty spectra $\sigma_{1}, \ldots, \sigma_{m+1}$ such that

$$
\left.\sigma_{1} \cup \sigma_{2} \cup \cdots \cup \sigma_{k} \subset \text { (int } \gamma_{1}\right) \cup\left(\text { int } \gamma_{2}\right) \cup \cdots \cup \text { (int } \gamma_{k} \text { ), }
$$

for $k=1,2, \ldots, m$, and $\bigvee_{h=1}^{m+1} \Re_{h}=\bigvee_{j=1}^{n} \Re_{j}$ (because each of the $\Re_{j}$ 's can be written as a finite direct sum of some of the $\tau_{h}$ 's).

It will be enough to prove that $\bigvee_{h=1}^{m+1} \Re_{h}=\bigoplus_{h=1}^{m+1} \Re_{h}$. In fact, if this last equality is true, then

$$
\bigvee_{j=1}^{n} \Re_{j}=\bigvee_{h=1}^{m+1} \Re_{h}=\bigoplus_{h=1}^{m+1} \Re_{h}=\bigoplus_{j=1}^{n} \Re_{j}=\Re_{0}
$$

each of the $\Re_{h}$ 's (and, a fortiori, each of the $\Re_{j}$ 's) is a hyperinvariant subspace of

$$
T\left|\mathscr{X}_{0}=\bigoplus_{h=1}^{m+1} T\right| \Re_{h}=\bigoplus_{j=1}^{n} T \mid \Re_{j}
$$


and

$$
\sigma\left(T \mid \mathscr{X}_{0}\right)=\bigcup_{h=1}^{m+1} \sigma_{h}=\bigcup_{j=1}^{n} \sigma\left(T \mid \Re_{j}\right)
$$

(see [2], [6], [10]).

We shall need a remarkable result due to David Hilbert ([7]; see also [12] for a generalization of this result, partially related with Lemma 7), which asserts that every closed rectifiable Jordan curve can be uniformly approximated by a sequence of lemniscates (i.e., level curves of polynomials). Since the distance from $\gamma_{h}$ to $\sigma$ is positive, it is clear that each of the curves $\gamma_{h}$ can be replaced by a lemniscate. In other words, we can directly assume that, for each $h=1, \ldots, m$, there exists a polynomial with complex coefficients, $p_{h}(z)$, such that $\gamma_{h}=\left\{z:\left|p_{h}(z)\right|=1\right\}$. Thus, if $m=1$ then the result follows immediately from Lemma 7 .

We shall proceed by induction on $m$. Let us assume that the result is true whenever the spectra can be separated by $m-1$ curves. This implies, in particular, that $\mathscr{X}_{1}=\bigvee_{h=1}^{m} \Re_{h}=\bigoplus_{h=1}^{m} \Re_{h}$, each of the $\Re_{h}$ 's, $h=$ $1, \ldots, m$, is hyperinvariant for $T\left|\mathscr{X}_{1}=\bigoplus_{h=1}^{m} T\right| \Re_{h}$ and $\sigma\left(T \mid X_{1}\right)=$ $\bigcup_{h=1}^{m} \sigma_{h}$. Therefore, $\mathscr{X}_{1}$ and $\Re_{m+1}$ are invariant subspaces of $T$ whose spectra $\sigma\left(T \mid \mathscr{X}_{1}\right)$ and $\sigma\left(T \mid \Re_{m+1}\right)=\sigma_{m+1}$ are separated by a single lemniscate $\gamma_{m}$. Hence, we can apply the results of Lemma 7 to this pair of subspaces to conclude that

$$
\mathscr{X}_{0}=\bigvee_{h=1}^{m+1} \Re_{h}=\mathscr{X}_{1} \vee \Re_{m+1}=\left(\bigoplus_{h=1}^{m} \Re_{h}\right) \oplus \Re_{m+1}=\bigoplus_{h=1}^{m+1} \Re_{h}=\bigoplus_{j=1}^{n} \Re_{j}
$$

is closed in $\mathscr{X}$ and $\sigma\left(T \mid \mathfrak{X}_{0}\right)=\sigma$. In particular, each of the subspaces $\mathfrak{T}_{j}$, $j=1, \ldots, n$, is hyperinvariant for $T \mid \chi_{0}$. The proof is now complete.

REMark. Example E shows that the hypothesis of Theorem 6 cannot be relaxed.

\section{REFERENCES}

1. T. Crimmins and P. Rosenthal, On the decomposition of invariant subspaces, Bull. Amer. Math. Soc. 73 (1967), 97-99.

2. R. G. Douglas and C. Pearcy, On a topology for invariant subspaces, J. Functional Analysis 2 (1968), 323-341.

3. P. R. Halmos, A Hilbert space problem book, Van Nostrand, Princeton, N.J., 1967.

4. H. Helson, Lectures on invariant subspaces, Academic Press, New York, 1964.

5. D. A. Herrero, On analytically invariant subspaces and spectra, Trans. Amer. Math. Soc. 233 (1977), 37-44.

6. D. A. Herrero and N. Salinas, Analytically invariant and bi-invariant subspaces, Trans. Amer. Math. Soc. 173 (1972), 117-136.

7. D. Hilbert, Uber die Entwicklung einer beliebigen analytischen Funktion einer Variablen in eine unendliche nach ganzen rationalen Funktionen fortschreitende Reihe, Gottinger Nachritten (1897), 63-70. 
8. T. B. Hoover, Quasi-similarity of operators, Illinois J. Math. 16 (1972), 678-686.

9. T. Kato, Perturbation theory for linear operators, Springer-Verlag, New York, 1966.

10. F. Riesz and B. Sz.-Nagy, Functional analysis, Ungar, New York, 1955.

11. J. D. Stafney, The spectrum of certain lower triangular matrices as operators on the $l_{p}$ spaces, Pacific J. Math. 42 (1972), 515-526.

12. J. L. Walsh and H. G. Russell, On the convergence and overconvergence of sequences of polynomials of best simultaneous approximation to several functions in distinct regions, Trans. Amer. Math. Soc. 36 (1934), 13-28.

Universidade Estadual de Campinas, IMECC, Campinas, SP, Brazil

Universidad Nacional de Rio IV ${ }^{\circ}$, Cordoba, Argentina

Current address: Department of Mathematics, IVIC, AP 1827, Caracas 101, Venezuela 